\title{
BMJ Open Qualitative evaluation of a complex intervention to implement health promotion activities according to healthcare attendees and health professionals: EIRA study (phase II)
}

\author{
Mariona Pons-Vigués, ${ }^{01,2,3}$ Anna Berenguera, ${ }^{01,2}$ Núria Coma-Auli, ${ }^{1}$ \\ Sebastià March, ${ }^{4}$ Haizea Pombo, ${ }^{5}$ Barbara Masluk, ${ }^{6}$ Montserrat Pulido-Fuentes, ${ }^{7}$ \\ Carmela Rodriguez, ${ }^{8}$ Juan A Bellón, ${ }^{9,10}$ Enriqueta Pujol-Ribera ${ }^{1,2,3}$
}

To cite: Pons-Vigués $\mathrm{M}$, Berenguera A, Coma-Auli N, et al. Qualitative evaluation of a complex intervention to implement health promotion activities according to healthcare attendees and health professionals: EIRA study (phase II). BMJ Open 2019;9:e023872. doi:10.1136/ bmjopen-2018-023872

- Prepublication history for this paper is available online To view these files, please visit the journal online (http://dx.doi org/10.1136/bmjopen-2018023872).

Received 2 May 2018

Revised 8 February 2019

Accepted 12 February 2019
Check for updates

(C) Author(s) (or their employer(s)) 2019. Re-use permitted under CC BY-NC. No commercial re-use. See rights and permissions. Published by BMJ.

For numbered affiliations see end of article.

Correspondence to Dr Mariona Pons-Vigués; mponsv@idiapjgol.info

\section{ABSTRACT}

Objective To evaluate the implementation and development of a complex intervention on health promotion and changes in health-promoting behaviours in primary healthcare according to healthcare attendees and health professionals. Design Descriptive qualitative evaluation research conducted with 94 informants. Data collection techniques consisted of 14 semistructured individual interviews, 9 discussion groups, 1 triangular group and 6 documents. Three analysts carried out a thematic content analysis with the support of Atlas.ti software. This evaluation was modelled on Proctor and colleagues' concept of outcomes for implementation research.

Setting 7 primary care centres from seven Spanish regions: Andalusia, Aragon, Balearic Islands, Basque Country, CastillaLa Mancha, Castilla-Leon and Catalonia.

Participants The study population were healthcare attendees (theoretical sampling) and health professionals (opportunistic sampling) who had participated in the exploratory trial of the EIRA intervention (2015).

Results Healthcare attendees and professionals had a positive perception of the study. Healthcare attendees even reported that they would recommend participation to family and friends. Health professionals became aware of the significance of the motivational interview, especially for health promotion, and emphasised social prescribing of physical activity. They also put forward recommendations to improve recruitment, screening and retention of participants. Healthcare attendees modified behaviours and health professionals modified working practices. To achieve sustainability, health professionals believe that it is crucial to adapt agendas and involve all the staff.

Conclusions The discourses of all stakeholders on the intervention must be taken into consideration for the successful, setting-specific implementation of adequate, acceptable, equitable and sustainable strategies aimed at health promotion and well-being.

\section{BACKGROUND}

Primary healthcare (PHC), the most accessible and most frequently used health service, provides comprehensive, long-term

\section{Strengths and limitations of this study}

- The sampling method of the qualitative evaluation might only have captured the experiences and views of the professionals and attendees more involved and positive with regard to the intervention and to health promotion.

- The rigour procedures applied (methodological adequacy, triangulation of techniques and analysis and reflexivity of the interdisciplinary research team) ensured the validity and reliability of the findings.

- The richness and complementarity of the information generated by healthcare attendees and health professionals from seven distinct regions will contribute to the adaptation of the intervention to the various settings to ultimately achieve feasible, sustainable integration in everyday primary care practice.

person-focused care. ${ }^{1}$ It is considered the ideal setting to implement individual, group and community health promotion interventions. However, these implementations face barriers and challenges set up by the system, the professionals and the public. ${ }^{23}$

Since it is very common for the same person to accumulate inter-related unhealthy behaviours, complex interventions are increasingly used in studies of behavioural change. In addition, first-hand knowledge of the setting where health promotion takes place is crucial when evaluating its effect. Complexity results from the number of interacting components, namely the amount and difficulty of behaviours required by those delivering or receiving the intervention, the number of groups or organisational levels targeted, the number and variability of outcomes and the degree of flexibility of the intervention. ${ }^{45}$ The main directives for 

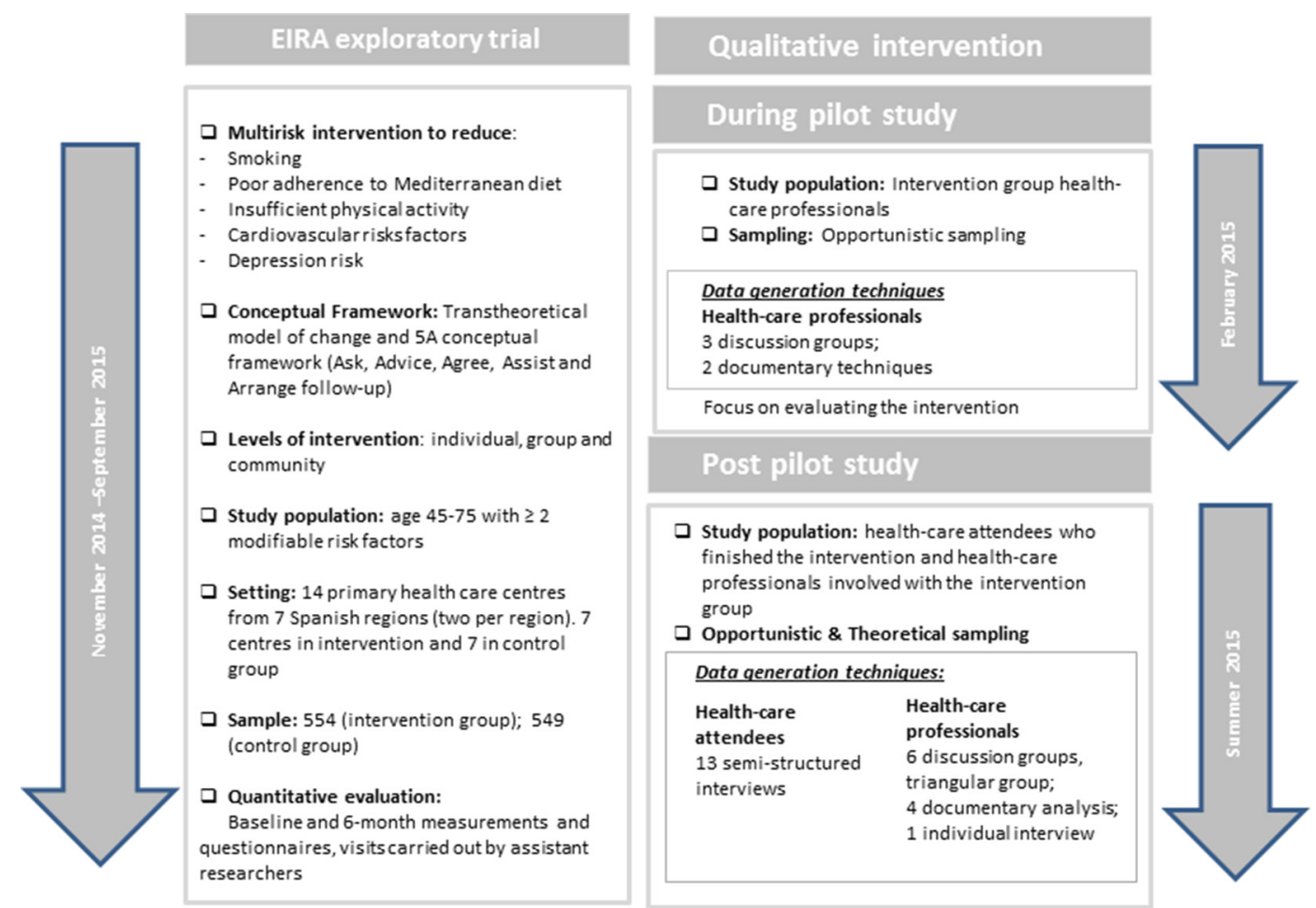

Figure 1 Second phase (development of an exploratory trial) of the EIRA Project, which follows the UK Medical Research Council framework for complex interventions.

the design, implementation and evaluation of complex interventions were developed by the Medical Research Council (MRC) ${ }^{467}$ using a mixed-method approach with five sequential phases: (1) definition of the theoretical foundation (preclinical phase), (2) construction of a model (phase I), (3) development of a pilot study (phase II), (4) completion of the definitive trial (phase III) and (5) long-term implementation (phase IV).

The EIRA Project started in Spain in 2012 with the objective to modify unhealthy behaviours in primary care patients following the MRC framework for complex interventions. ${ }^{67}$ To date, the first three phases have been completed. ${ }^{238-10}$ Specifically, the objective of the EIRA Project was to design, conduct and evaluate a complex, multirisk intervention to enhance adherence to the Mediterranean diet, increase insufficient physical activity and reduce smoking, cardiovascular risk factors and risk of depression in people aged 45-75 years that contact PHC services with at least two of these behaviours or risk factors. Participants receive individual recommendations on their behaviour and risk factors, and they are offered to attend group sessions and social prescription of health promoting community assets. The person-centred approach uses the motivational interview, and the attendee becomes an active agent in her own life. Participants allocated to the control group receive the usual care (figure 1).

A key question in evaluating a complex intervention is actual effectiveness. However, the process itself is also important: what happens, how, when and why. The process evaluation in trials explores the implementation of an intervention, assesses its quality and fidelity, clarifies causal mechanisms and identifies contextual factors associated with variation in outcomes. ${ }^{41}$ Qualitative methodology has a unique role in understanding the implementation process of an intervention ${ }^{12}$ : interestingly, qualitative research can be used concurrently with a pilot trial, for instance, to optimise recruitment and informed consent strategies, to identify acceptability of the intervention, to provide insights into processes of change and to help interpret findings. ${ }^{13}$ Accordingly, the qualitative evaluation of the intervention implementation process is able to identify determinants of clinical practice such as barriers and facilitators that influence the adoption of organisational and professional change. ${ }^{14}$ This qualitative evaluation facilitates understanding of how and why the different components of the intervention are successfully or unsuccessfully implemented; it also contributes to identify predictive factors of success and generates useful knowledge for advancing the implementation of scientific evidence. ${ }^{15}$ In addition, the qualitative methodological perspective might transcend the main limitations of the quantitative approach that prevails in clinical trials and provides essential information on the evaluation of interventions, since it involves the different stakeholders, which actively convey their experiences, opinions, needs and suggestions for improvement.

This qualitative evaluation presents the results of the second phase (development of an exploratory trial) of the EIRA Project. The objective was to evaluate: (1) the process of implementation and development of a complex intervention on health promotion in primary 
care according to healthcare attendees and health professionals and (2) changes in health-promoting behaviours.

\section{METHODS \\ Design}

Descriptive qualitative research based on the experiences of participants was used to evaluate the exploratory trial of the EIRA complex intervention.

\section{Setting and study population}

Seven primary care centres (PCCs) included in the intervention group of the EIRA Project from seven Spanish regions (one PCC per region) participated: Andalusia, Aragon, Balearic Islands, Basque Country, Castilla-La Mancha, Castilla-Leon and Catalonia. The control group of the exploratory trial did not participate in the qualitative evaluation.

The study population were: (A) PHC professionals from participating PCC (including family physicians, primary care nurses, social workers and administrative staff) and assistant researchers (in charge of performing baseline and 6-month measurements and questionnaires); and (B) healthcare attendees aged 45-75 years who participated and completed the EIRA study.

\section{Sample design and participant selection strategy}

PHC professionals from participating PCC and assistant researchers were selected by means of opportunistic sampling. ${ }^{16}$ The site investigator of each PCC contacted all professionals who participated in the EIRA study to book group interviews 2-3 months after the beginning of recruitment (February 2015 in three centres) and at the end of the intervention (summer of 2015 in the seven centres of the intervention group). The decision of PHC professionals to participate in the group interviews was voluntary. For healthcare attendees, we applied theoretical sampling based on a prior definition of participants' characteristics to obtain optimal variety and discursive wealth. ${ }^{16}$ Fifteen informant profiles emerged from the discursive variants sex, age, educational level and type of intervention (the approach to the first component of the intervention was decided by the participant). Next, two of these profiles were randomly allocated to each PCC included in the intervention group of the EIRA Project; one PCC had three profiles. At the end of the intervention (summer 2015), the site investigator of each PCC contacted by phone the healthcare attendees participating in the EIRA Project who met the specific informant profile for the PCC to explain the objectives of the qualitative evaluation and invited them to participate in an interview. The voluntary aspect of participation was also emphasised to healthcare attendees.

\section{Data collection and generation techniques}

Conversational techniques were used for PHC professionals: three discussion groups in February 2015 and six discussion groups at the end of the intervention, in the summer of 2015; one triangular group (a meeting of three people to discuss together a topic or issue with the aim of ascertaining the range and intensity of their views $)^{17}$; and one individual interview with a community agent. In addition, we collected the written reports of six professionals who could not attend the discussion groups because of scheduling conflict (two documentary techniques in February and four in summer). Table 1 details the main characteristics of the 81 PHC professionals who participated in the study.

Semistructured individual interviews were used to collect information from healthcare attendees. We initially planned a semistructured individual interview for each of the 15 profiles of informant; however, two semistructured individual interviews could not take place because the participants could not be contacted after the end of the study. We finally held 13 interviews with healthcare attendees. Table 2 shows the characteristics of these 13 participants.

In total, data collection techniques consisted of 14 semistructured individual interviews, 9 discussion groups, 1 triangular group and 6 documents. Semistructured individual interviews, discussions groups and triangular group followed a topic guide with open-end questions, with some adaptations according to type of informant and study period (box 1). The topic guides were based on a review of the literature and the objectives of the study. After obtaining informed consent from the participants, all interviews were audio or audio and video recorded. The discussion groups took place in the PCC with one moderator and one observer and lasted between $90 \mathrm{~min}$ and $120 \mathrm{~min}$. Semistructured individual interviews took place in a setting accessible for the healthcare attendees and lasted between $15 \mathrm{~min}$ and $60 \mathrm{~min}$. The field work was carried out in each region by qualified interviewers with experience in qualitative research. Informative richness for a deeper understanding of the development and implementation of the intervention was achieved.

\section{Data analysis}

All interviews and discussion groups were transcribed verbatim and deidentified by trained personnel. ${ }^{18} \mathrm{~A}$ thematic content analysis was carried out ${ }^{19}{ }^{20}$ with the support of Atlas.ti software. The data were analysed as follows by three researchers (NCA, MPV and EPR, who are a nurse, a pharmacist and a physician, respectively): (1) formulation of preanalytical intuitions after successive readings of the transcriptions and the notes from documentary techniques; (2) creation of an initial analytical plan and text codification; (3) creation of categories by grouping the codes according to the analogy criterion based on Proctor and colleagues' model of outcomes for implementation research ${ }^{21}$ and new elements from the discourses; (4) analysis of each category and relationship with the others; and (5) elaboration of the new text with the main results. These results were presented and discussed in a meeting with all research members of the EIRA Project (January 2016). 


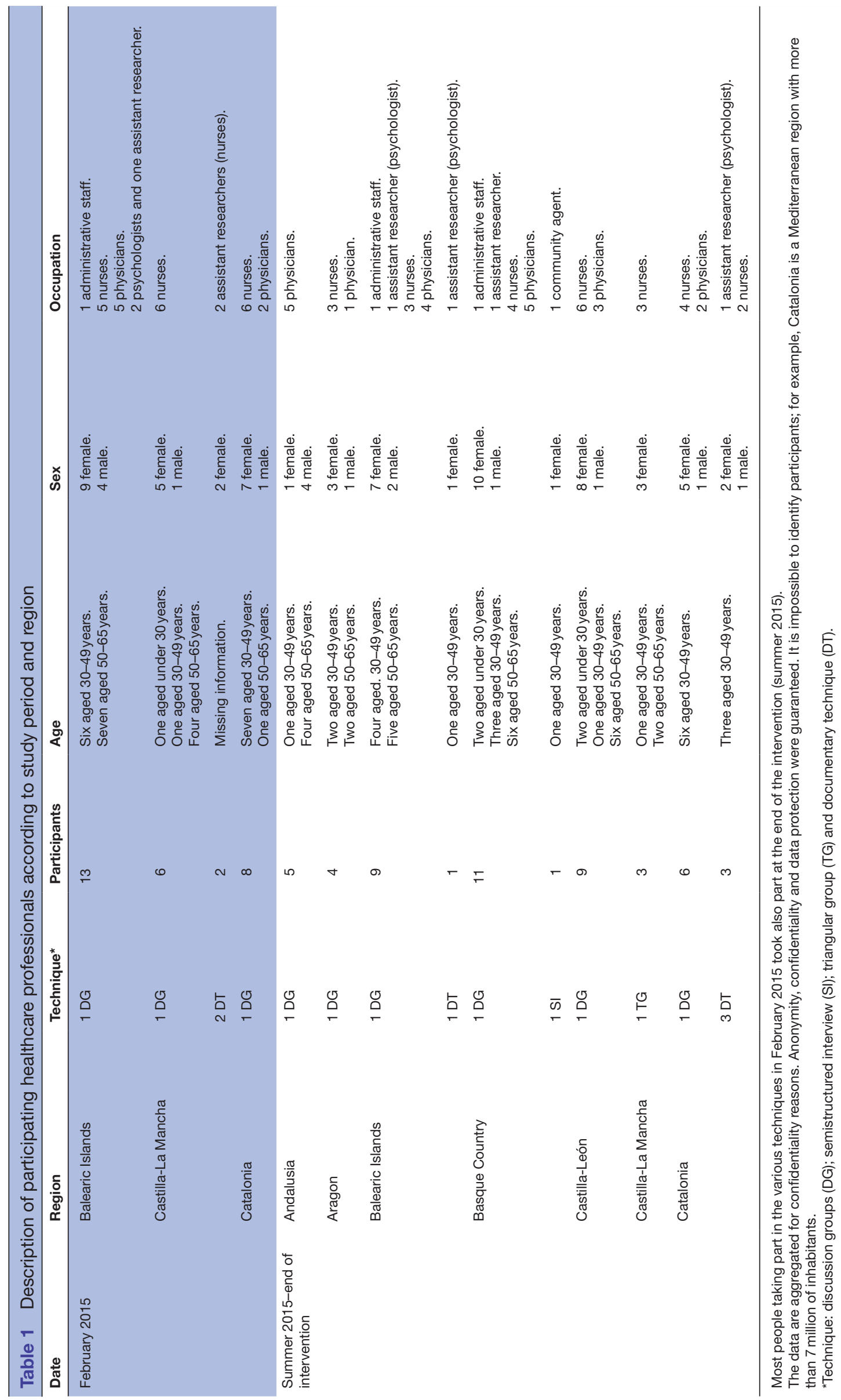


Table 2 Description of participant healthcare attendees by region (summer 2015, end of the intervention)

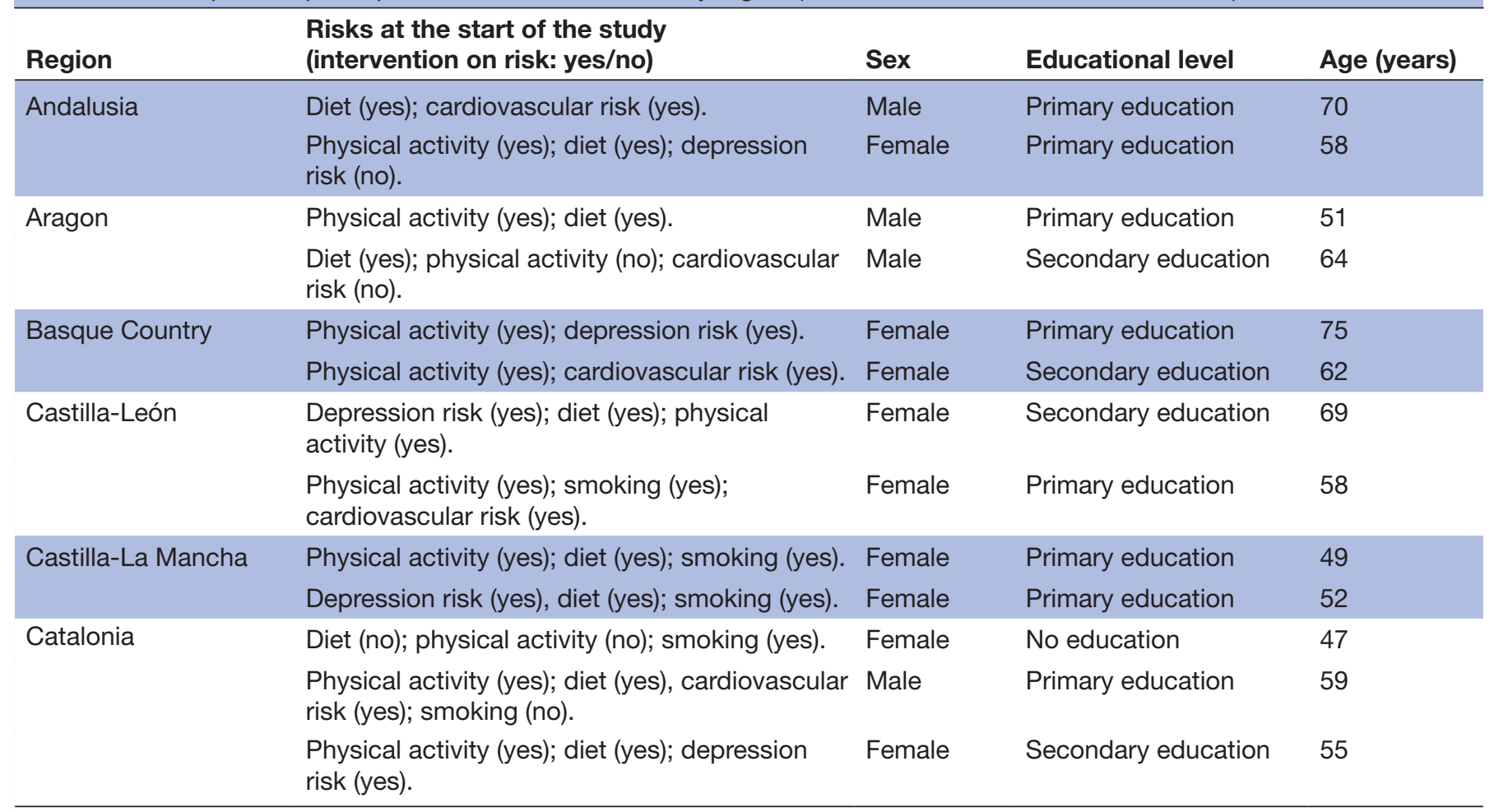

No semistructured interviews with healthcare attendees took place in the Balearic Islands.

Anonymity, confidentiality and data protection were guaranteed. It is impossible to identify participants; for example, Catalonia is a Mediterranean region with more than 7 million of inhabitants.

\section{Rigour and quality criteria}

To guarantee quality and rigour, we adhered to the following recommendations ${ }^{22}{ }^{23}$ : description of the intervention, the context, the participants and the research process; methodological adequacy; working with different actors; triangulation of techniques (comparison of data obtained by means of different information collection techniques) and analysis (contrasting and comparing the data analyses performed by different analysts to strengthen the credibility and confirmability of the study results); and reflexivity of the interdisciplinary research team. Sufficient data were collected to meaningfully answer the research question. The authors guarantee the accuracy, transparency and honesty of the data and information contained in the study.

\section{Ethical considerations}

This study followed the tenets of the Declaration of Helsinki. All participants signed the informed consent form. Anonymity, confidentiality and data protection were guaranteed.

\section{Patient and public involvement}

Study participants were not involved in the development of the research question or the outcome measures nor the design of the study. The results will be presented to study participants and citizens through informative activities and the media.

\section{RESULTS}

The results are classified in five categories: acceptability, appropriateness and feasibility, sustainability, penetration (changes implemented) and suggestions for improvement. Table 3 shows the definitions of these categories complemented with illustrative quotations from the discussions.

\section{Acceptability}

In general, healthcare attendees and health professionals reported satisfaction with their participation, and their final evaluation was positive. Healthcare attendees described being thankful to the professionals for their support, and they explained that they felt more confident making decisions about the process of change. All healthcare attendees interviewed would recommend participating in the study to family and friends, and in fact some had already done it. They affirmed that participation requires being ready to pay attention, to listen and to reflect.

Health professionals believed in health promotion and while they did not consider the contents of the intervention innovative, they indicated that it changes working practices, notably the systematisation of recommendations and the boost of social prescription. However, they remained critical and underscored that the project was too ambitious, too long, somehow unclear and unorganised, which led to confusion during implementation. 
Box 1 Topic guide for the data generation techniques based on type of informant and study period

\section{Primary healthcare professionals and assistant researchers (February 2015)}

- We will start talking about recruitment: in your experience, what do you suggest to enhance recruitment?

- Based on your experience, how can we improve the suggested screening strategy?

- What is your experience with the algorithm of prioritisation and what do you suggest to improve it?

- What is your overall assessment of the proposed intervention?

-What do you suggest to improve the feasibility of each component of the intervention (diet, physical activity, smoking, cardiovascular risk and depression risk)?

- What are your views and experiences on the resources and materials of the intervention (web for professionals, web for patients, patient information leaflets, SMS other ICTs)?

- How does the target population accept each aspect of the study (how do they feel, ask and talk about their difficulties with regard to behavioural change)?

- How could we boost participation in the study (consent at recruitment, follow-up ...)?

What do you suggest to improve the coordination of the project?

Finally, how do you perceive your participation in this project?

\section{Primary healthcare professionals and assistant researchers (summer 2015)}

- What is your overall assessment of the intervention?

- Has your participation in this study been useful to modify any aspect of clinical practice? Do you think that it has been useful for patients?

- What are your suggestions to improve the feasibility of each component of the intervention?

- Concerning each component of the intervention: would you keep them in the definitive trial? Would you keep them at each level of intervention? How does the population accept them? (How do they feel, ask and talk about their difficulties with regard to behavioural change?)

- What are your views on the relevance and usefulness of the resources and materials of the intervention (web for professionals, web for patients, patient information leaflets, SMS and other Information and communications technology - ICTs-)?

- How could we improve the coordination of the project?

- How do you assess the feasibility of expanding this project to other primary care centres?

- How do you evaluate your participation in this project?

- Finally, do you have any comment on recruitment, screening strategy and prioritisation algorithm?

\section{Healthcare attendees (summer 2015)}

- Could you please explain your overall experience with the EIRA study?

- Which activities have you carried out during your participation in the study? (If none, ask about group interventions and social prescription.)

- Do you think that you have participated in decision making about your own health? How was your experience?

- Has this study contributed to adopt healthier behaviours? Has any aspect of your life changed since you entered the study? Do you think that it is feasible to integrate the recommendations and activities suggested by primary care professionals into your daily life?

Continued

\section{Box 1 Continued}

- Have you found useful the resources related to the EIRA Project such as the webpage, SMS and so on?

-What could we improve?

- Would you recommend participation in a similar study to your family and friends?

- How do you evaluate your participation in the EIRA Project?

They specifically highlighted difficulties in the approach to risk of depression. Moreover, in some primary care teams tension emerged between professionals that participated and their non-participating colleagues.

\section{Appropriateness and feasibility}

The results have been categorised according to the phases of the study.

\section{Training}

Although some professionals considered that the training conducted prior intervention was appropriate and provided new concepts, they maintained that it was insufficient for the actual implementation of the intervention, specifically concerning the motivational interview and the approach to risk of depression. There was no practical training in the use of online case report forms (CRF), and in one of the centres, the training was provided too early. Some theoretical aspects could not be translated into practice due to lack of time or skills.

\section{Coordination}

With regard to coordination, the professionals found the meetings with the research team useful. However, it was sometimes unclear how to proceed, how to give appointments and refer healthcare attendees for follow-up or who was responsible for reviewing the study tests. In addition, some procedures were changed after the start of the study. Reiteration of questions and lost to follow-up were generated by the complexity of circuits and the lack of communication between professionals.

\section{Recruitment}

Most professionals agreed that recruitment involved a higher workload than anticipated and that it took place in a short timeframe. They explained that it was difficult to explain the study and to encourage healthcare attendees to participate, and they believed that many enrolled because they felt commitment to their professionals. Healthcare attendees explained that they participated because they thought it was interesting, they had time and they felt commitment to their regular health professionals.

Professionals pointed at a selection bias caused by the recruitment of frequent attenders, patients generally better controlled and more motivated. No random systematic sampling was applied, and any reason for consultation was accepted. Several professionals from the selected PCC declined participation. 


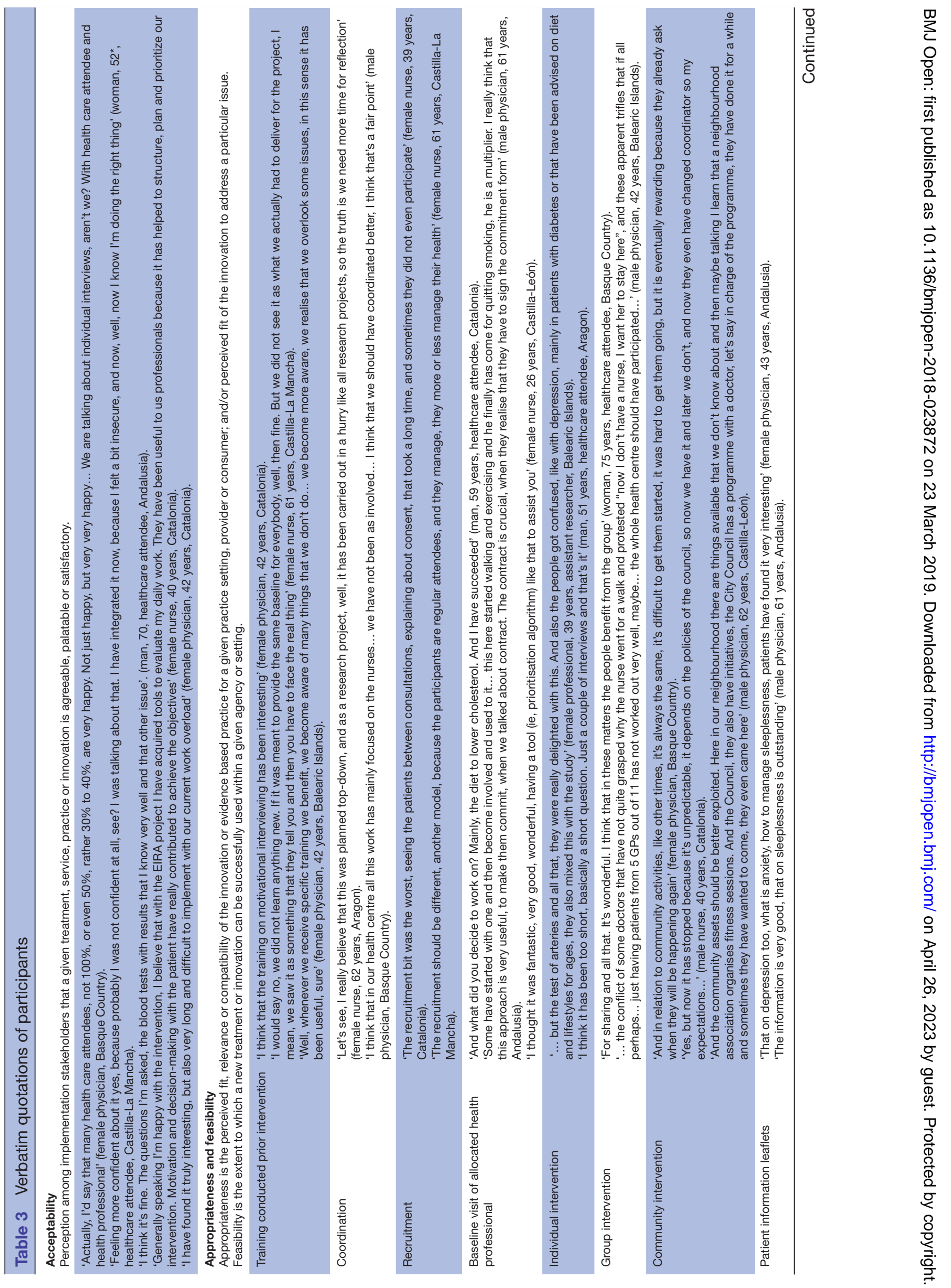




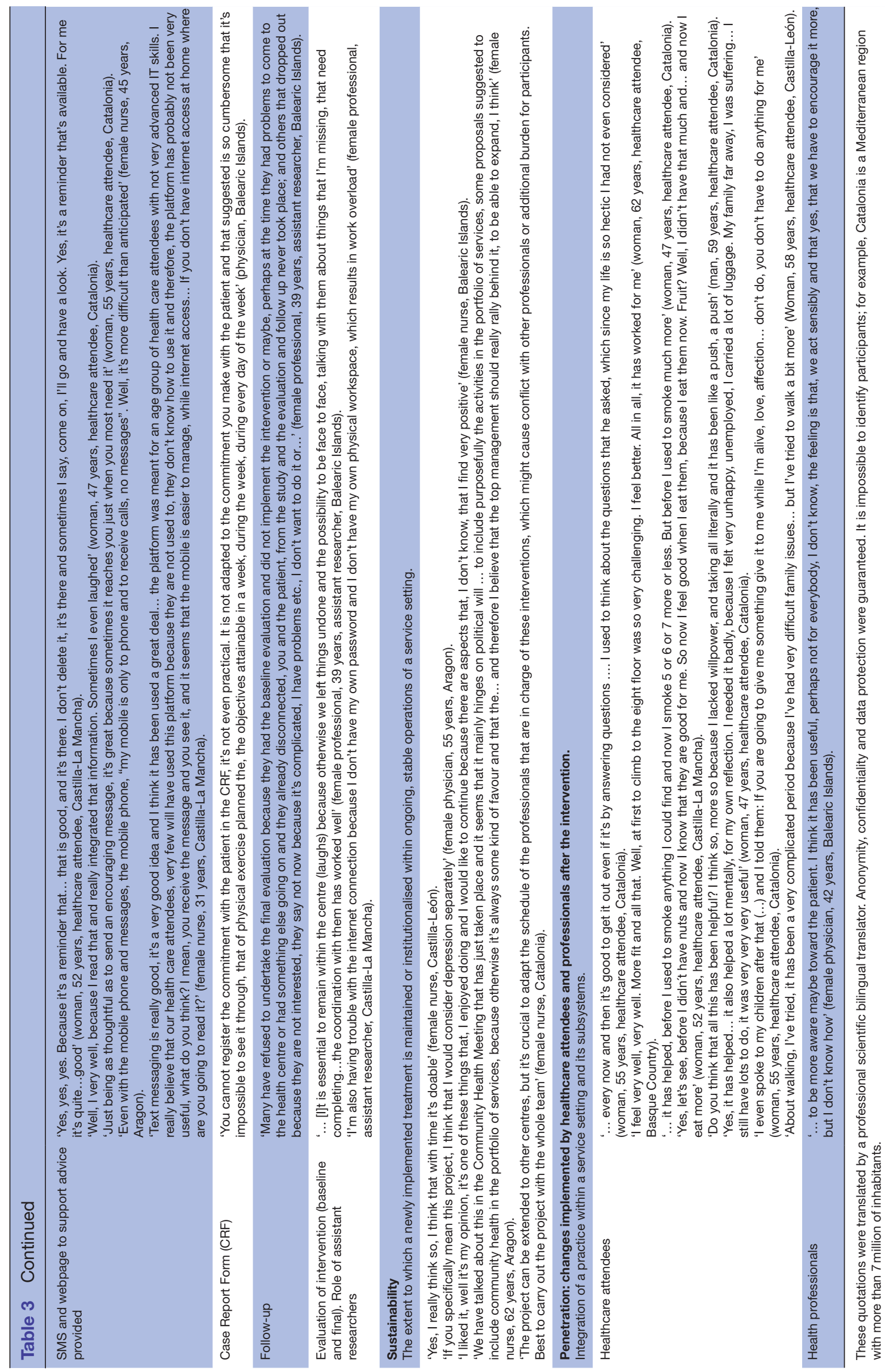


In particular, few admission staff chose to take part, and their involvement was often hurried and uncoordinated, which increased the workload of the professionals involved in the study.

First visit with the health professional (prioritisation of behaviours to modify and intervention plan)

Most healthcare attendees evaluated positively their involvement in decision making and many explained that they participated in the prioritisation of behaviours and risks that needed changing. Patients asserted that trust in the health professional facilitates change. Health professionals evaluated positively the patients' assessment of their own risk behaviours followed by the decision about which behaviours to modify. Professionals also indicated that the prioritisation algorithm was useful.

\section{Individual intervention}

Healthcare attendees believed that the advice was useful and applicable, and they felt that health professionals really cared and listened to them. They emphasised that in comparison with usual visits health professionals had more time to attend to them without rush and to do a holistic valuation. The healthcare attendees that received health promotion recommendations in regular practice mixed them up with the intervention advice of the study. They also mixed up the clinical intervention with the collection of information for the clinical trial. They thought that the follow-up period should be extended and include more people. The professionals were positive about the person-centred approach and have become more aware of the significance of the motivational interview and of health promotion.

\section{Group intervention}

Group activities focused on physical activity and nutrition. Healthcare attendees explained that sharing experiences was positive; they established new relationships and organised walking groups. Some professionals reported that these activities are difficult to implement due to lack of time. For others, these activities do not fall within their duties (they considered them additional activities or simply going for a stroll with healthcare attendees).

\section{Community intervention}

Although few centres used activities already popular in the neighbourhood, social prescription was very positively evaluated both by healthcare attendees and professionals. Most physical activities prescribed were organised by the town councils. For professionals, social prescription was a novel concept, and they emphasised that adherence is unknown since attendance was not registered.

\section{Health education leaflets}

Healthcare attenders favoured personal contact over patient information leaflets. However, the few comments received on leaflets are all positive, especially those about diet or mental health. Healthcare professionals considered that the leaflets were a useful tool, particularly regarding diet, and even patients who did not participate in the study received them. They also believed that healthcare attendees appreciate written information.

\section{SMS and health education webpage}

Few healthcare attendees agreed to receive SMS, but those that accepted explained that SMS were helpful and encouraging. Professionals considered SMS useful reminders. The webpage was hardly accessed, for which healthcare attendees and professionals provided various reasons: lack of recommendation, no access to computers/ Internet, lack of motivation and feeling uncomfortable sitting in front of a screen.

\section{Online CRF}

The professionals believed that the study online CRF was too complicated, too slow and that it was difficult to register personalised agreements. Also, since the programme was separate from the electronic health records, they had to work with both programmes simultaneously. In addition, poor internet connection slowed the work of some professionals.

\section{Follow-up}

Health professionals indicated that follow-up data such as adherence rates were somewhat unclear and would be interested in learning about the final results. They believed that retention of participants might be determined by difficulties in attending the intervention visits, loss of interest and the perception that no added value is attached to these interventions.

\section{Evaluation of the intervention (baseline and final): role of the}

\section{assistant researchers}

Generally, healthcare attendees evaluated positively the questionnaires and tests carried out by the assistant researchers (blood tests, evaluation of vascular health and so on) because they felt listened to and had more time to talk. The professionals believed that healthcare attendees felt well cared for because they spent sufficient time with the interviews. The assistant researchers indicated that they had to administer too many questionnaires. They also pointed at the following issues: insufficient information, lack of their own working space, irregular access to the CRF and lack of authorisation to consult the medical history of healthcare attendees.

\section{Sustainability}

Some professionals considered that it is important to extend this intervention to other PCC but underscored the need for the support of institutions, for extended consultation length and the involvement of all professionals. In addition, risk of depression remains a controversial component of the intervention. Some professionals would exclude it altogether, while others believed that it needs a different approach. 
Penetration: changes implemented by healthcare attendees and professionals after the intervention

Healthcare attendees reported increased motivation and knowledge of healthy behaviours and feeling more positive towards change. Those working with physical activity and nutrition explained that they implemented changes and described high levels of satisfaction: they walked more, got less tired and felt fitter, ate healthier (smaller amounts, more vegetables, fruit and nuts and use of olive oil) and some stated that they drank less alcohol. They also stated that they smoked less cigarettes. Professionals agreed that healthcare attendees made an effort to meet their objectives, to implement changes and to start healthier habits.

The barriers for change according to healthcare attendees were: family responsibilities (care of the sick, care of grandchildren, house chores and so on), life-work imbalance, weather conditions and lack of willpower. The professionals agreed with these barriers and added financial issues and unawareness of the need to change. Facilitators of change according to healthcare attendees were: group activities and trust in health professionals. For health professionals, the healthcare attendees should decide which behaviours to modify because their commitment implies autonomy and empowerment and facilitates change.

The professionals reflected on how to approach health promotion in primary care: with a holistic view of healthcare attendees, providing evidence-based advice, being more purposeful, using motivational interview, involving the family and prioritising social prescription. Participation in the intervention facilitated a deeper knowledge of healthcare attendees and extended consultation length. Professionals reported improvement in the assessment and register of activities in the electronic health records.

\section{Suggestions for improvement}

Table 4 shows the discourses and suggestions for improvement of participants.

\section{DISCUSSION}

Overall, health professionals and healthcare attendees shared a positive perception of their participation in the study. Indeed, healthcare attendees would even recommend it to family and friends. Health professionals realised the significance of the motivational interview, in particular with regard to health promotion. They also underscored the potential of social prescribing in relation to physical activity. In addition, health professionals put forward suggestions to improve recruitment, screening and retention of participants. Healthcare attendees modified behaviours and health professionals revised working practices. According to health professionals, the continuity of this programme is contingent on adapting agendas and involving all staff.

We regard the positive attitude of healthcare attendees and health professionals towards this health promotion multibehavioural intervention as an endorsement of the definitive trial of the EIRA Project. However, we acknowledge that the current version of this intervention cannot yet be integrated in primary care practice until fundamental organisational changes that ensure feasibility and sustainability in real-world conditions take place. Even though the intervention was adapted and implemented following the recommendations of healthcare attendees and health professionals obtained in prior phases of the EIRA Project, ${ }^{2}{ }^{38-10}$ further adjustments are required. For instance, in the EIRA Project, we concluded that for health promotion, it is essential to involve most primary care professionals, including administrative staff, to avoid tension and to challenge the notion that health promotion is voluntary or based on personal preference. It is also important to reduce the work overload (objectively high), to simplify recruitment and screening questionnaires and to modify the approach to emotional discomfort and risk of depression. However, it is crucial to participate in the dissemination of social prescription and to continue the research in implementation strategies focusing on equity and on improving overall results. It has also been observed that primary care professionals require more resources, time, skills and motivation to reach out and work with the community in health promotion. ${ }^{24}$

Healthcare attendees reported high levels of satisfaction with the study because they felt that professionals gave them enough time and listened to their needs and preferences. They also felt supported during the process of change and were able to initiate sustainable healthy behaviours. We might thus conclude that the intervention encouraged a holistic, person-centred approach underscoring the key role of the primary care professional and of the motivational interview as a useful strategy to promote behavioural change. ${ }^{25}$ The motivational interview requires training and extended consultation times, ${ }^{26}$ and although health professionals received basic training (4hours at the beginning of the study), most agree that further training is required.

Although some health professionals underscored the pivotal role of PHC to manage risk of depression, many worried about lack of skills, attitudes, tools and experience, in agreement with others authors. ${ }^{27}$ In addition, some healthcare attendees had a positive opinion about the opportunity to know their depression risk. ${ }^{28}$ To some extent, the recommendations to manage emotional discomfort in primary care take all these views into account. ${ }^{29}$ The real objective of the first approach is to ascertain the nature of the emotional discomfort by means of active listening, probing and empathy to understand the meaning, adaptability and problem solving skills of each person to avoid chronification and medicalisation.

This project encourages participation in community activities, particularly physical activity, even though many participants did not follow these recommendations. In agreement with the results of the systematic review by March $e t a l$, which shows that in primary care preventive interventions, the community might be more effective 







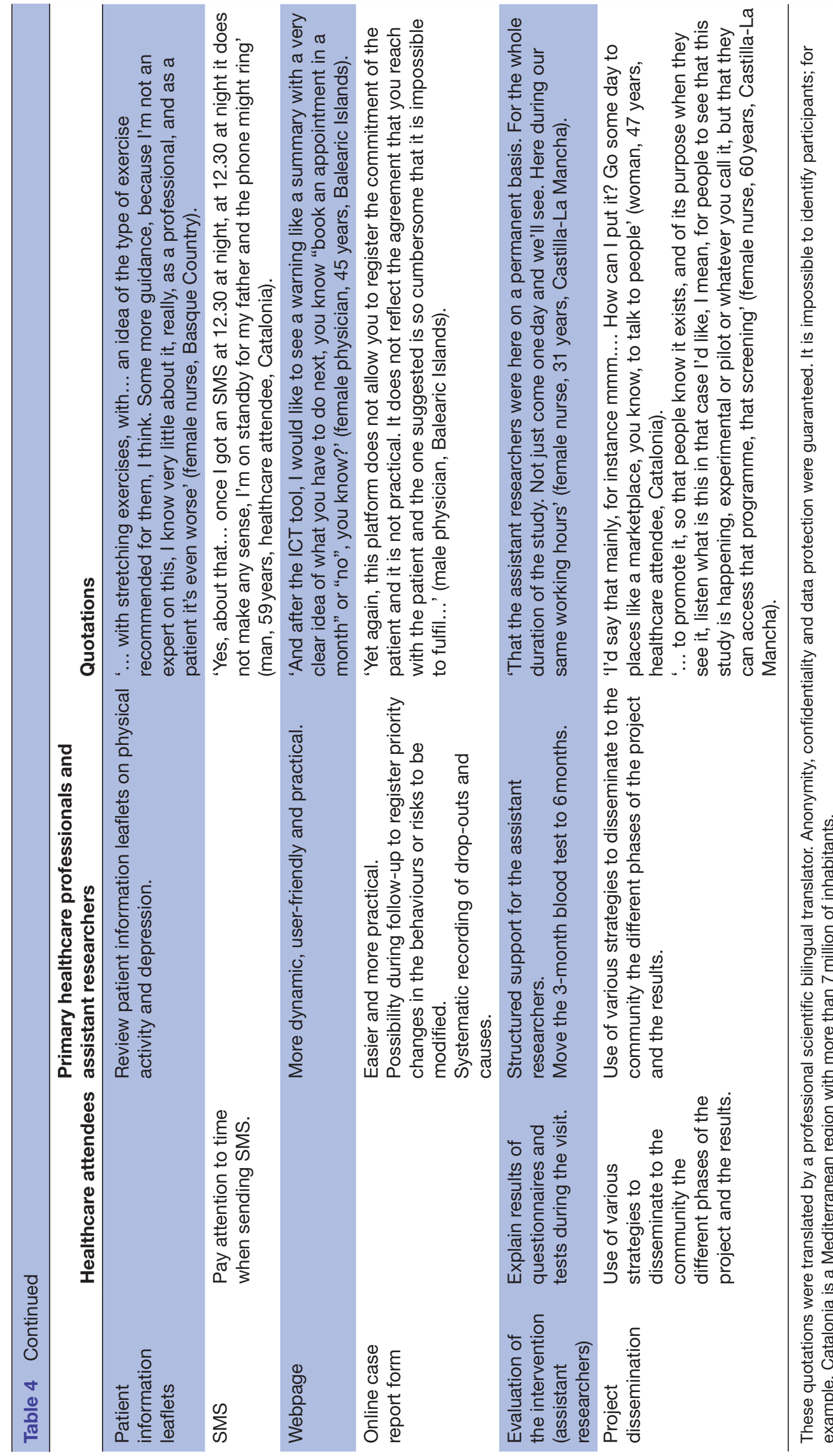


than the individual approach, ${ }^{30}$ health professionals underscored a more systematic use of social prescription in regular practice, which indicates an interest in implementing a more biopsychosocial model. ${ }^{31}$ This qualitative assessment suggests that despite early resistance, professionals and healthcare attendees became eventually aware of the importance of the community components of health promotion interventions. In addition, the feasibility of community recommendations is suggested as a selection criteria of PCC with capability to develop complex health promotion interventions based on networks that identify, promote and evaluate local health assets. ${ }^{32}$ In contrast, despite the growing holistic, psychological and collective conception of health, ${ }^{9}$ the persistence of the biomedical paradigm is shown by the positive evaluation of medical tests by healthcare attendees.

Most professionals and healthcare attendees considered SMS, a low cost method that preserves privacy, useful for people with mobile phones. This outcome is consistent with other studies that suggest that SMS are effective in health promotion interventions, particularly regarding quitting smoking and physical activity, where SMS can be used to provide positive feedback in order to effect and maintain behavioural change. ${ }^{33-35}$ In contrast, the webpage was not considered useful for participants, in agreement with other studies that stress the relevance of the patient-health professional relationship. ${ }^{35} 36$

\section{Limitations and strengths}

In the EIRA Project, healthcare attendees and health professionals provided information and were consulted about the development and evaluation of the intervention. However, further steps towards deeper changes in research practice should involve more effective participation in decision making. ${ }^{37}$

Despite the use of theoretical sampling for healthcare attendees, the voice of participants with higher education qualifications (only $6 \%$ in the pilot trial) was insufficient. Also, the voluntary character of participation of healthcare attendees and professionals in this qualitative evaluation might imply that only the experiences and opinions of people with a positive view of the intervention and of health promotion were collected. However, the detailed description of less successful aspects, the polarisation of professionals regarding the benefits of the study and the suggestions for improvement point at a diversity of standpoints. We believe that it is nonetheless fundamental to add the perspective of less motivated professionals and of participants that dropped out or that simply decided not join the study. Although participants of this qualitative study and of the EIRA Project comprise people from various geographical origins, the contribution of particularly vulnerable individuals (female carers, immigrants and people with precarious employment) remains inadequate. This subpopulation probably lack sufficient time and need more attention regarding health promoting behaviour. More research is needed to further understanding of vulnerable patients.
One strength of the study is the use of the MRC approach for the design, implementation and evaluation of complex interventions. ${ }^{467}$ The following phase of the intervention (definitive trial) will more specifically adapt to the people and setting and will be more sustainable thanks to the richness and complementarity of the information generated by healthcare attendees and professionals from these seven regions. The evaluation process was also analysed by quantitative methods (paper under construction), but considering the limited sample of the pilot trial and the low response rate to questionnaires, qualitative evaluation has proven crucial to understand how healthcare attendees and professionals perceive the intervention. Moreover, the rigour procedures applied ensured the validity and reliability of the findings. Although the authors of the current evaluation are also members of the EIRA research team, positive and negative information on the intervention was rigorously collected to deepen understanding on the components that need improvement (see table 4).

\section{CONCLUSIONS}

The discourses of all stakeholders with regard to the intervention must be taken into consideration for a successful, setting-specific implementation of the most adequate, acceptable, equitable and sustainable strategies for health promotion and well-being.

\section{Author affiliations}

${ }^{1}$ Fundació Institut Universitari per a la recerca a I'Atenció Primària de Salut Jordi Gol i Gurina (IDIAPJGol), Barcelona, Spain

${ }^{2}$ Universitat Autònoma de Barcelona, Barcelona, Spain

${ }^{3}$ Departament d'Infermeria, Universitat de Girona, Girona, Spain

${ }^{4}$ Primary Care Research Unit of Mallorca, Balearic Health Services-IbSalut, Palma, Spain

${ }^{5}$ Primary Care Research Unit of Bizkaia, Basque Health Service-0sakidetza, Bilbao, Spain

${ }^{6}$ Psychology and Sociology Department, University of Zaragoza, Zaragoza, Spain ${ }^{7}$ Facultad de Terapia Ocupacional, Logopedia y Enfermería, Universidad de CastillaLa Mancha, Talavera de la Reina, Spain

${ }^{8}$ Primary Care Research Unit, The Alamedilla Health Centre, Castilla and León Health Service (SACYL), Biomedical Research Institute of Salamanca (IBSAL), Salamanca,

Spain

${ }^{9}$ Health District of Primary Care Málaga-Guadalhorce, SAS, Málaga, Spain

${ }^{10}$ Biomedical Research Institute of Malaga (IBIMA), Málaga, Spain

Acknowledgements We are grateful to all healthcare attendees and professionals who participated in the study and shared their experiences. We would also like to thank Anna Moleras, Bonaventura Bolíbar, Joan Llobera, José Ángel Maderuelo, Rosa Magallón, Vicente Martínez, Elena Melús, Emma Motrico, Fernando Salcedo, Marc Casajuana, Edurne Zabaleta and Tomas López for their contributions and Eulàlia Farré for the English translation.

Contributors MP-V, $A B$ and EP-R designed the study and wrote the protocol. EP-R, MP-V, AB, BM, MP-F, SM, HP, CR and JAB participated in data collection and generation techniques. MP-V, NC-A and EP-R conducted the analysis. All authors contributed to the interpretation of results. MP-V, AB and EP-R wrote the first draft of the manuscript. All authors read, contributed and approved the final version of the manuscript.

Funding This project has been funded by the Carlos III Health Institute (Ministry of Economy and Competitiveness, Spain) with a grant for Research Projects on Health (PI12/01914; Pl12/001616; Pl12/02608; Pl12/01974; PI12/02774; PI12/02635; PI12/02379; and P15/00114) through the Network for Prevention and Health 
Promotion in Primary Care (redlAPP, RD12/0005/0001; RD16/0007/0001) and by European Union European Regional Development Fund (ERDF) funds (European Regional Development Fund).

Competing interests None declared.

Patient consent for publication Obtained.

Ethics approval The study was approved by the Research Ethics Committee of the IDIAP Jordi Gol (2013; P12/073). The authors guarantee the accuracy, transparency and honesty of the data and information contained in the study.

Provenance and peer review Not commissioned; externally peer reviewed.

Data sharing statement № additional data are available.

Open access This is an open access article distributed in accordance with the Creative Commons Attribution Non Commercial (CC BY-NC 4.0) license, which permits others to distribute, remix, adapt, build upon this work non-commercially, and license their derivative works on different terms, provided the original work is properly cited, appropriate credit is given, any changes made indicated, and the use is non-commercial. See: http://creativecommons.org/licenses/by-nc/4.0/.

\section{REFERENCES}

1. Starfield B, Shi L, Macinko J. Contribution of primary care to health systems and health. Milbank Q 2005;83:457-502.

2. Rubio-Valera M, Pons-Vigués M, Martínez-Andrés M, et al. Barriers and facilitators for the implementation of primary prevention and health promotion activities in primary care: a synthesis through metaethnography. PLoS One 2014;9:e89554.

3. Moreno-Peral P, Conejo-Cerón S, Fernández A, et al. Primary care patients' perspectives of barriers and enablers of primary prevention and health promotion-a meta-ethnographic synthesis. PLoS One 2015;10:e0125004.

4. Craig P, Dieppe P, Macintyre S, et al. Developing and evaluating complex interventions: the new Medical Research Council guidance. BMJ 2008;337:a1655.

5. Petticrew M. When are complex interventions 'complex'? When are simple interventions 'simple'? Eur J Public Health 2011;21:397-8.

6. Campbell M, Fitzpatrick R, Haines A, et al. Framework for design and evaluation of complex interventions to improve health. BMJ 2000;321:694-6.

7. Campbell NC, Murray E, Darbyshire J, et al. Designing and evaluating complex interventions to improve health care. BMJ 2007;334:455-9.

8. Zabaleta-del-Olmo E, Bolibar B, García-Ortíz L, et al. Building interventions in primary health care for long-term effectiveness in health promotion and disease prevention. A focus on complex and multi-risk interventions. Prev Med 2015;76:S1-4.

9. Pons-Vigués M, Berenguera A, Coma-Auli N, et al. Health-care users, key community informants and primary health care workers' views on health, health promotion, health assets and deficits: qualitative study in seven Spanish regions. Int J Equity Health 2017;16:99.

10. Berenguera A, Pons-Vigués M, Moreno-Peral P, et al. Beyond the consultation room: proposals to approach health promotion in primary care according to health-care users, key community informants and primary care centre workers. Health Expect 2017;20:896-910.

11. Moore GF, Audrey S, Barker M, et al. Process evaluation of complex interventions: medical research council guidance. BMJ 2015;350:h1258.

12. Ludvigsen MS, Meyer G, Hall E, et al. Development of clinically meaningful complex interventions - the contribution of qualitative research. Pflege 2013;26:207-14.

13. Cooper C, O'Cathain A, Hind D, et al. Conducting qualitative research within clinical trials units: avoiding potential pitfalls. Contemp Clin Trials 2014;38:338-43.

14. Nilsen P. Making sense of implementation theories, models and frameworks. Implement Sci 2015;10:53.
15. Palinkas LA, Aarons GA, Horwitz S, et al. Mixed method designs in implementation research. Adm Policy Ment Health 2011;38:44-53.

16. Moser A, Korstjens I. Series: Practical guidance to qualitative research. Part 3: Sampling, data collection and analysis. Eur J Gen Pract 2018;24:9-18.

17. Conde F. Los grupos triangulares como espacios transicionales para la producción discursiva. In: Huelva L, ed. Culturas e identidades urbanas. Sevilla: Junta de Andalucia-Fundación El Monte, 1996:275-307.

18. MacLean LM, Meyer M, Estable A. Improving accuracy of transcripts in qualitative research. Qual Health Res 2004;14:113-23.

19. Morse JM, Richards L. Readme first for a user's guide to qualitative methods: Sage, 2002.

20. Pope C, Ziebland S, Mays N. Qualitative research in health care. Analysing qualitative data. BMJ 2000;320:114-6.

21. Proctor E, Silmere H, Raghavan R, et al. Outcomes for implementation research: conceptual distinctions, measurement challenges, and research agenda. Adm Policy Ment Health 2011;38:65-76.

22. Tuckett AG. Part II. rigour in qualitative research: complexities and solutions. Nurse Res 2005;13:29-42.

23. Calderón C. Evaluación de la calidad de la investigación cualitativa en salud: criterios, proceso y escritura. Forum Qual Sozialforsch / Forum Qual Soc Res 2009;10.

24. Cabeza E, March S, Cabezas C, et al. [Healthcare promotion in primary care: if Hippocrates were alive today...]. Gac Sanit 2016;30 Suppl 1:81-6.

25. Miller WR, William R, Rollnick S. Motivational interviewing: helping people change: Guilford Press, 2013.

26. Söderlund LL, Madson MB, Rubak S, et al. A systematic review of motivational interviewing training for general health care practitioners. Patient Educ Couns 2011;84:16-26.

27. Dowrick C, May C, Richardson M, et al. The biopsychosocial model of general practice: rhetoric or reality? Br J Gen Pract 1996;46:105-7.

28. Bellón JÁ, Moreno-Peral P, Moreno-Küstner B, et al. Patients' opinions about knowing their risk for depression and what to do about it. The predictD-qualitative study. PLoS One 2014;9:e92008.

29. Castelló M, Fernández de Sanmamed MJ, García J, et al. Atenció A les persones amb malestar emocional relacionat amb condicionants socials a l' atenció primària de salut. Barcelona: Fòrum Català d'Atenció Primària, 2016.

30. March S, Torres E, Ramos M, et al. Adult community healthpromoting interventions in primary health care: a systematic review. Prev Med 2015;76:S94-104

31. March S, Ripoll J, Jordan Martin M, et al. Factors related to the development of health-promoting community activities in Spanish primary healthcare: two case-control studies. BMJ Open 2017;7:e015934.

32. Cofiño R, Aviñó D, Benedé CB, et al. [Health promotion based on assets: how to work with this perspective in local interventions?]. Gac Sanit 2016;30(Suppl 1):93-8.

33. Hall AK, Cole-Lewis H, Bernhardt JM. Mobile text messaging for health: a systematic review of reviews. Annu Rev Public Health 2015;36:393-415.

34. Whittaker R, McRobbie H, Bullen C, et al. Cochrane Tobacco Addiction Group. Mobile phone-based interventions for smoking cessation. Cochrane Database Syst Rev 2016;17.

35. Berenguera A, Molló-Inesta À, Mata-Cases M, et al. Understanding the physical, social, and emotional experiences of people with uncontrolled Type 2 diabetes: a qualitative study. Patient Prefer Adherence 2016;10:2323-32.

36. Carmona-Terés V, Moix-Queraltó J, Pujol-Ribera E, et al. Understanding knee osteoarthritis from the patients' perspective: a qualitative study. BMC Musculoskelet Disord 2017;18:225.

37. Boote J, Baird W, Beecroft C. Public involvement at the design stage of primary health research: a narrative review of case examples. Health Policy 2010;95:10-23. 